\title{
De Novo Design of Antiviral and Antibacterial Peptides with Varying Loop Structures
}

\author{
Guangshun Wang $^{1 *}$, Karen W. Buckheit ${ }^{2}$, Biswajit Mishra1, Tamara Lushnikova1, and Robert W. Buckheit Jr. ${ }^{2}$ \\ ${ }^{1}$ Department of Pathology and Microbiology, University of Nebraska Medical Center, 986495 Nebraska Medical Center, Omaha, NE 68198-6495, USA \\ ${ }^{2}$ ImQuest BioSciences, Inc., 7340 Executive Way, Suite R, Frederick, MD 21704, USA
}

\begin{abstract}
Although the rate of new HIV infections has been declining, AIDS continues to be one of the leading causes of death worldwide. The lack of an effective HIV vaccine makes it necessary to develop alternative strategies, such as the development of topical microbicides, to prevent transmission. Antimicrobial peptides represent promising microbicide candidates. Previously, we succeeded in enhancing the anti-HIV activities of several peptides that form helical structures based on the bioinformatic results learned from the antimicrobial peptide database. This study showed that Lys-to-Arg alterations also improved the HIV inhibitory activity of thanatin which is known to form a $\beta$-hairpin structure. Using a previously reported de novo designed HIV inhibitory peptide GLR-19 as the starting template, loop structures of varying sizes were generated by restraining a disulfide bond at different positions. The thanatin-mimicking constructs are referred to as GLRC peptides since they are composed of only four amino acid residues $G, L, R$, and $C$. While GLRC-2, the peptide with a medium-sized loop structure, was most potent against HIV-1 and HSV-2, GLRC-3, with the small loop structure, was most potent against Escherichia coli K12. Thus, the efficacy of the GLRC peptides is microbe dependent. Further terminal sequence truncation of GLRC-2 reduced antimicrobial activity against both viruses and bacteria. It appears that the high antiviral potency of GLRC-2 is related to high hydrophobicity, although a wide-range correlation is lacking. In addition, GLRC-2, which is more active against viruses, is also more resistant to the action of chymotrypsin. Therefore, GLRC-2, a novel peptide that acquired not only higher stability but also higher anti-HIV activity than the GLR-19 template, serves as the starting point for additional rounds of peptide engineering.
\end{abstract}

Keywords: Antimicrobial peptides; HIV-1; Peptide stability; Microbicides

\section{Introduction}

Since the report of the first human immunodeficiency virus (HIV) case in 1981, the acquired immunodeficiency syndrome (AIDS) has become the fourth leading cause of death worldwide. The United Nations estimates that there are now 40 million people living with HIV/ AIDS. Thus, it is urgent to develop novel therapeutic and preventative agents to reduce the transmission of HIV. Because the majority of HIV infections are acquired through heterosexual intercourse, it would be helpful if measures can be taken to stop or prevent the sexual transmission of HIV $[1,2]$.

We have been focusing on identifying potent anti-HIV candidates derived from naturally occurring antimicrobial peptides (AMPs). These peptides have been proven to have antimicrobial effects on a broad range of bacteria, viruses, fungi, and parasites [3-6]. Select amphibian and piscine AMPs are known to reduce infectivity of channel catfish virus (CCV), frog virus 3 (FV3), and HIV [7,8]. In addition, AMPs with anti-HIV activity have also been discovered from other sources, which include melittin and cecropin from insects [9], cathelicidins and defensins from mammals [5,10-13], and cyclotides (i.e. circular peptides) from plants [14]. We previously identified the most potent as well as the minimal anti-HIV fragments within human cathelicidin LL37 [13]. A follow-up study showed that LL-37 fragments inhibited HIV reverse transcriptase in vitro [15]. It is also likely that the antiviral effect of vitamin $\mathrm{D}$ is associated with the expression of human LL-37 in vivo [16]. We also found that BMAP-18, a fragment derived from bovine cathelicidin BMAP-27, is a HIV-1 inhibitor $($ EC50 $=0.83 \mu \mathrm{g} / \mathrm{ml})$ with a therapeutic index (TI) of 24 [13]. To identify additional templates, we have evaluated 30 peptides selected from the antimicrobial peptide database $[17,18]$. The results of that peptide screening led to the identification of $10 \mathrm{HIV}$ inhibitory peptides with EC50 values in the range of 1.0 to $10 \mu \mathrm{g} / \mathrm{ml}$ [19]. This study extends the results of our previous studies by evaluating AMPs with different loop structures. These peptides were designed using GLR-19 as a template. GLR-19 was previously de novo designed based on the antimicrobial peptide database $[17,19]$. We also compared the stability of these peptide scaffolds to the action of chymotrypsin. The results of the current study are reported herein.

\section{Materials and Methods}

\section{Peptides}

All the peptides were chemically synthesized using the solid-phase method and purified by HPLC to >95\% (Genemed Synthesis, Inc., TX).

\section{Anti-HIV assays}

Anti-HIV-1 cytopathic effect (CPE)-inhibition assays were performed as described [20]. In brief, serially diluted peptides were added to a 96-well round bottom microtiter plate in triplicate. CEM-

*Corresponding author: Guangshun Wang, Department of Pathology and Microbiology, University of Nebraska Medical Center, 986495 Nebraska Medical Center, Omaha, NE 68198-6495, USA, Tel: (402) 559-4176; Fax: (402) 559-4077; E-mail: gwang@unmc.edu

Received October 13, 2011; Accepted November 05, 2011; Published December 10, 2011

Citation: Wang G, Buckheit KW, Mishra B, Lushnikova T, Buckheit RW (2011) De Novo Design of Antiviral and Antibacterial Peptides with Varying Loop Structures. J AIDS Clinic Res S2:003. doi:10.4172/2155-6113.S2-003

Copyright: @ 2011 Wang G, et al. This is an open-access article distributed under the terms of the Creative Commons Attribution License, which permits unrestricted use, distribution, and reproduction in any medium, provided the original author and source are credited. 
SS cells at a concentration of $2.5 \times 10^{3}$ cells per well and HIV-1IIIB at the appropriate pre-determined titer were sequentially added to the microtiter plate. The cultures were incubated at $5 \% \mathrm{CO} 2 / 37^{\circ} \mathrm{C}$ for six days. Following the incubation, the microtiter plates were stained with XTT tetrazolium dye to evaluate the efficacy and toxicity of the test compound(s). Using 4-parameter curve fitting, values of EC50 (50\% inhibition of virus replication), and $\mathrm{TC}_{50}(50 \%$ reduction in cell viability), as well as a therapeutic index $\left(\mathrm{TI}=\mathrm{TC}_{50} / \mathrm{EC}_{50}\right)$, were determined for each peptide.

\section{Antibacterial assays}

The antibacterial activity of the peptides was analyzed using the standard approach of microdilution [21]. The bacterial strains used were Escherichia coli $\mathrm{K} 12$ and Bacillus subtilis. In brief, small bacterial cultures were grown overnight. Fresh cultures were inoculated with a small aliquot of the overnight culture and incubated at $37 \mathrm{oC}$ until the optical density A600 of the culture reached 0.5 . The bacterial culture (90 $\mu \mathrm{l}$ each) was then diluted to an A600 of 0.001 and partitioned into a 96-well plate with $\sim 10^{5}$ colony-forming units (CFU) per well. The cells were then treated with $10 \mu \mathrm{l}$ of the peptide at different concentrations, allowing the minimum inhibitory concentration (MIC) measurement for each. The assay was duplicated and repeated multiple times. The plate was then further incubated at $37 \mathrm{oC}$ overnight $(\sim 16 \mathrm{hrs})$ and read at $630 \mathrm{~nm}$ on a ChroMate 4300 microplate reader (GMI, MN).

\section{High performance liquid chromatography (HPLC)}

The retention time of the peptide was measured on a Waters HPLC system equipped with an analytical reverse-phase Waters symmetry C8 column $(150 \times 3.9 \mathrm{~mm})$. The peptide was eluted by a gradient of acetonitrile (containing 1\% TFA) from $5 \%$ to $95 \%$ at a flow rate of $1 \mathrm{ml} /$ min. The peptide peak was detected by UV at $254 \mathrm{~nm}$.

\section{Electrophoresis assays of peptide stability}

Peptide (0.5 mM) and chymotrypsin (molar ratio 40:1) were mixed and incubated at $37 \mathrm{oC}$ in $10 \mathrm{mM}$ PBS buffer ( $\mathrm{pH} 8.0$ ). An aliquot $(20 \mu \mathrm{l})$ of the reaction was taken at different hours and immediately mixed with $10 \mu \mathrm{l}$ of SDS loading buffer to terminate the action of the protease. Aliquots of the reactions were subsequently analyzed using $18 \%$ stacking tricine SDS-PAGE. The bands were stained by Coomassie Brilliant Blue R250 (Fisher). Images of the gels were taken and digitized in Photoshop 6.0 for band quantification.

\section{Results}

\section{Engineering of HIV inhibitory peptides based on an AMP} with a b-sheet structure

Our previous studies focused on linear peptides from which several HIV inhibitory candidates have been identified. Those peptides such as the GI-20 and BMAP-18 have a tendency to form amphipathic helical structures, especially when in complex with membranes [2123]. In this study, BAMP-18 was included as a control and it showed a similar efficacy in Table 1 as reported previously [13]. To expand the conformation space, we shifted our focus to AMPs with a $\mu$-sheet structure. Thanatin is a natural AMP isolated from the insect Podisus maculiventris (i.e., the spined soldier bug) [24]. This peptide was chosen because it has a simple hairpin structure with a single disulfide bond (C11-C18) between the two strands (Figure 1A) [25]. Two derivatives of thanatin were obtained. In the structure-based peptide 1 (SB1), the last residue Met of thanatin was substituted with Ile to reduce possible oxidation of the peptide. This peptide demonstrated activity against HIV-1 but poor activity against HSV-2 (Table 1). To enhance the anti-HIV activity of SB1, residues K3, K4, and K17 were converted to arginines based on previous findings from the AMP database $[17,18]$. SB2, the arginine analog of SB1, indeed showed higher activity against HIV-1. However, these changes deprived SB1 of the weak activity against HSV-2 (Table 1). Since there was no increase in cytotoxicity, the reduced EC50 value of SB2 directly translated into a greater TI (Table 1 ). The TI of SB2 reached 30 , which is slightly greater than those ( $\mathrm{TI}=21-25)$ determined for GI-20 (derived from human LL-37), BMAP-18 (derived from cattle cathelicidin BMAP-27), and an arginine analog of dermaseptin S9 [13,19].

\section{De novo design of peptides with various loop structures}

We designed additional peptides using GLR-19 as the starting sequence. This peptide is a de novo designed peptide which is known to be inhibitory to HIV-1 $[17,19]$. We found that GLR-19 also showed antiviral activity against HSV-2 (Table 1). To mimic the hairpin structure of thanatin, we introduced a disulfide bond at different positions within GLR-19 (Figure 1). In each case, the incorporation of a disulfide bond into GLR-19 was at the expense of two leucines, which were replaced by cysteines. Initially, a disulfide bond (yellow lines in Figure 1) was positioned between residues 2 and 18 (C2-C18) to generate a large loop structure. This disulfide bond was shifted to generate additional peptides with a reduced loop size (Figure 1). We referred to these disulfide bond-linked peptides as GLRC peptides since their sequences are composed of only $G, L, R$ and $C$ amino acid residues. GLRC-1, the peptide with the large 17-residue loop, yielded an EC50 greater than $100 \mu \mathrm{g} / \mathrm{ml}$, which was the highest concentration evaluated in antiviral assays [13]. GLRC-2, however, the peptide with a middle-sized 13-residue loop, demonstrated the highest potency $(\mathrm{EC} 50=1.81 \mu \mathrm{g} / \mathrm{ml})$. When the size of the loop was furthered reduced to obtain GLRC-3, antiviral activity was reduced. In parallel antiviral assays, GLRC-2 also showed the best activity against HSV-2 compared

A

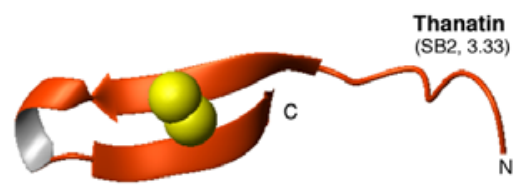

B

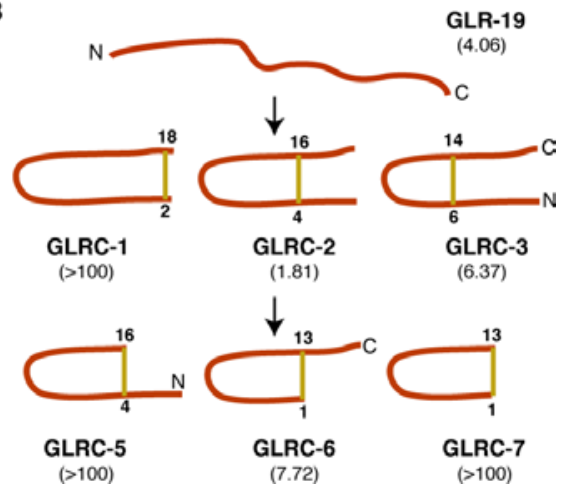

Figure 1: De novo design of novel anti-HIV peptides by mimicking the $\beta$-hairpin structure of thanatin. (A) A ribbon view of the 3D structure of thanatin (PDB ID: 8TFV) [25]. The disulfide bond is represented by a pair of yellow balls. (B) Systematic construction of peptides with varying loops or scaffolds. The starting template, GLR-19, consists of only three frequently occurring residues found in natural antimicrobial peptides collected in the antimicrobial peptide database $[17,19]$. For additional details on peptide design, see the text. Yellow bars represent a disulfide bond and the peptide chain in orange. The anti-HIV activity for each peptide fold (i.e. the EC50 value from Table 1 ) is given in parenthesis. 


\begin{tabular}{|c|c|c|c|c|c|c|c|c|c|}
\hline \multirow{2}{*}{ Peptide } & \multirow[b]{2}{*}{$\begin{array}{l}\text { Amino acid } \\
\text { sequence }\end{array}$} & \multirow{2}{*}{$\begin{array}{l}\# \text { of } \\
\text { AA }\end{array}$} & \multirow[b]{2}{*}{ S-S bond } & \multicolumn{3}{|c|}{ HIV-1IIIB in CEM-SS cells } & \multicolumn{3}{|c|}{ HSV-2MS in Vero cells } \\
\hline & & & & $\begin{array}{l}E_{50}{ }^{a} \\
(\mu \mathrm{g} / \mathrm{ml})\end{array}$ & $\begin{array}{l}\mathrm{TC}_{50} \\
(\mu \mathrm{g} / \mathrm{ml})\end{array}$ & TI & $\begin{array}{l}\mathrm{EC}_{50} \\
(\mu \mathrm{g} / \mathrm{ml})\end{array}$ & $\begin{array}{l}\mathrm{TC}_{50} \\
(\mu \mathrm{g} / \mathrm{ml})\end{array}$ & TI \\
\hline BMAP-18 & $\begin{array}{l}\text { GRFKRFRKK } \\
\text { FKKLFKKIS }\end{array}$ & 18 & & 0.83 & 19.8 & 23.9 & 27.2 & $>100$ & $>3.7$ \\
\hline SB1 & $\begin{array}{l}\text { GSKKPVPIIYC } \\
\text { NRRTGKCQRI }\end{array}$ & 21 & C11-C18 & 13.1 & $>100$ & $>7.6$ & 64.9 & $>100$ & $>1.5$ \\
\hline SB2 & $\begin{array}{l}\text { GSRRPVPIIYC } \\
\text { NRRTGRCQRI }\end{array}$ & 21 & C11-C18 & 3.33 & $>100$ & $>30$ & $>100$ & $>100$ & --- \\
\hline GLK-19 & $\begin{array}{l}\text { GLKKLLGKLL } \\
\text { KKLGKLLLK }\end{array}$ & 19 & & $>47.5$ & 25.1 & $<0.5$ & n.e. ${ }^{b}$ & n.e. & \\
\hline GLR-19 & $\begin{array}{l}\text { GLRRLLGRLL } \\
\text { RRLGRLLLR }\end{array}$ & 19 & & 4.06 & 17.8 & 4.4 & 2.32 & 12.4 & 5.3 \\
\hline GLRC-1 & GCRRLLGRLLRRLGRLLCR & 19 & C2-C18 & $>100$ & 59.2 & $<1.0$ & 30 & $>100$ & $>3.3$ \\
\hline GLRC-2 & GLRCRLGRLLRRLGRCLLR & 19 & C4-C16 & 1.81 & 17.8 & 9.83 & 5.43 & 31.8 & 5.9 \\
\hline GLRC-3 & GLRRLCGRLGRRLCRLLLR & 19 & C6-C14 & 6.37 & 18.5 & 2.9 & 15 & 62.3 & 4.2 \\
\hline GLRC-4 & GCRRLCGRLGRRLCRLLCR & 19 & $\begin{array}{l}\text { C2-C18 } \\
\text { C6-C14 }\end{array}$ & 69.3 & $>100$ & $>1.44$ & 64.7 & $>100$ & $>1.5$ \\
\hline GLRC-5 & GLRCRLGRLLRRLGRC & 16 & C4-C16 & $>100$ & 59.5 & $<1.00$ & n.e. & n.e. & \\
\hline GLRC-6 & CRLGRLLRRLGRCLLR & 16 & C1-C13 & 7.72 & 41.9 & 5.43 & n.e. & n.e. & \\
\hline GLRC-7 & CRLGRLLRRLGRC & 13 & $\mathrm{C} 1-\mathrm{C} 13$ & $>100$ & $>100$ & --- & n.e. & n.e. & \\
\hline GLRC-8 & GLRCLRLRGRLRLGRCLLR & 19 & C4-C16 & $>100$ & 31 & $<1.00$ & n.e. & n.e. & \\
\hline
\end{tabular}

Table 1: Antiviral activities of de novo designed peptides. aAlthough not provided in the table, the standard error from multiple antiviral assays is on average less than $10 \%$ of the respective mean EC50 or TC50 values. bn.e., not evaluated.

\begin{tabular}{|c|c|c|c|c|c|c|c|}
\hline $\begin{array}{l}\text { Peptide } \\
\text { name }\end{array}$ & $\begin{array}{l}\text { Net } \\
\text { charge }\end{array}$ & $\begin{array}{l}\text { \# of } \\
\mathrm{R} \text { or } \mathrm{K}\end{array}$ & Pho\% & $\begin{array}{l}\text { HPLC } \\
t_{R}(\min )\end{array}$ & $\begin{array}{l}\text { Boman } \\
\text { index }\end{array}$ & $\begin{array}{l}\text { E. coli } \\
\text { MIC }(\mu \mathrm{M})\end{array}$ & $\begin{array}{l}\text { B. subtilis } \\
\mathrm{MIC}(\mu \mathrm{M})\end{array}$ \\
\hline GLK-19 & 7 & $7 \mathrm{~K}$ & 47 & 9.776 & -0.43 & $10^{a}$ & n.e. ${ }^{b}$ \\
\hline GLR-19 & 7 & $7 R$ & 47 & 9.263 & 3.01 & $>120$ & n.e. \\
\hline GLRC-1 & 7 & $7 R$ & 47 & 7.452 & 3.4 & 30 & $>120$ \\
\hline GLRC-2 & 7 & $7 \mathrm{R}$ & 47 & 12.344 & 3.4 & 30 & $>120$ \\
\hline GLRC-3 & 7 & $7 \mathrm{R}$ & 47 & 10.101 & 3.4 & 7.5 & $>120$ \\
\hline GLRC-4 & 7 & $7 R$ & 47 & 9.601 & 3.78 & 60 & $>120$ \\
\hline GLRC-5 & 7 & $6 \mathrm{R}$ & 43 & 9.373 & 3.39 & 60 & $>120$ \\
\hline GLRC-6 & 7 & $6 \mathrm{R}$ & 50 & 9.395 & 3.47 & 30 & 60 \\
\hline GLRC-7 & 6 & $5 R$ & 46 & 8.734 & 3.88 & 120 & $>120$ \\
\hline GLRC-8 & 8 & $7 R$ & 47 & 9.18 & 3.4 & $15-30$ & $>120$ \\
\hline
\end{tabular}

Table 2: Effects of peptide scaffolds on their bacterial inhibitory activities. a. Data were taken from ref. [19]. bn.e., not evaluated.

to both GLRC-1 and GLRC-3 (Table 1). These results highlight the importance of the C4-C16 disulfide linkage in GLRC-2. Consistent with this observation, GLRC- 4 was also determined to be inactive when two different disulfide bonds (C2-C18 and C6-C14) were applied in the peptide. However, the GLRC peptides with disulfide bonds all had reduced activity against HSV-2 compared to the linear template GLR19 (Table 1). The possibility that linear AMPs are generally more active than their disulfide counterparts against HSV-2 remains to be tested.

Next, we further mimicked the scaffold of SB2 by removing either the $\mathrm{N}$ - or the C-terminal residues outside of the loop (Figure 1). When the C-terminal segment LLR was truncated, the resulting peptide GLRC-5 became inactive. In contrast, the peptide GLRC-6 remained active after the N-terminally exposed residues, GLR, of GLRC-2 were removed. Another peptide (GLRC-7) was obtained by removing both the $\mathrm{N}$ - and C-terminal segments outside of the loop of GLRC2. Like GLRC-5, GLRC-7 was inactive. These results suggest that the C-terminal LLR segment of GLRC-2 is important for anti-HIV activity. We also evaluated if the sequence of the central fragment between the two Cys residues of GLRC-2 played a role in antiviral activity. To address this question, we altered the sequence order without changing the amino acid composition. To our surprise, the resulting peptide GLRC-8 was found to be inactive (Table 2). We conclude that both the order of the central sequence and the C-terminal region of GLRC-2 play an important role in HIV inhibition.

Because these GLRC peptides are novel, we also evaluated their antibacterial activity using representative laboratory strains. Overall, these peptides showed poor activity against Gram-positive B. subtilis (Table 2). In the case of Gram-negative E. coli K12, the most active peptide ( $\mathrm{MIC}=7.5 \mu \mathrm{M}$ ) was GLRC-3, the peptide with the smallest loop (Figure 1). Peptides GLRC-1, GLRC-2, GLRC-6, and GLRC-8 showed similar anti-E. coli activity $(\mathrm{MIC}=30 \mu \mathrm{M})$, indicating that the N-terminal segment GLR of GLRC-2 is dispensable for antibacterial activity. Interestingly, the least active peptide was determined to be GLRC-7, the shortest GLRC peptide with a 13-residue loop structure. Thus, the C-terminal segment of GLRC-2 appears to be important also for bactericidal activity.

\section{Peptide parameters that influence activity}

To provide further insight into the antimicrobial activities of these peptide constructs, we measured the retention times of the GLRC peptides by reverse-phase HPLC (Table 2). The retention time is related to peptide hydrophobicity [23]. Other parameters such as net charge, hydrophobic residues\% (pho\% in Table 3), and Boman index [18] (i.e. protein binding potential) [3] were also calculated for the 
GRLC peptides using the prediction interface of the AMP database (http://aps.unmc.edu/AP/main.html) [18] and are included in Table 3. While it is not obvious why GRLC-3 is most active against E. coli, the high potency of GLRC-2 against HIV-1 is likely to be due to high hydrophobicity of the peptide, which has the longest retention time (tR) (Table 2).

\section{Stability of the GLRC peptides to chymotrypsin}

We also compared the stability of the GLRC peptides to the action of proteases such as chymotrypsin. Figure 2 shows the percentage of intact peptide after protease treatment at $37 \mathrm{oC}$ for 4 hrs. For comparison, SB1 was also tested and was found to have $23 \%$ intact peptide remaining at $4 \mathrm{hrs}$. Compared to SB1, the linear template GLR-19, with $15.5 \%$ intact peptide remaining, was less stable (Figure 2). Restraining the GLR-19 polypeptide chain with disulfide bonds had a significant impact on peptide stability. First, we determined that peptide stability was related to the loop size: GLRC-2 > GLRC-1 > GLRC-3 (Figure 1). Thus, GLRC-2, with a medium-sized loop structure, acquired improved stability ( $>60 \%$ intact peptide) compared to SB1 (23\% intact peptide, Figure 2). The stability of GLRC-2 increased by 4.4 fold relative to GLR-19. Note that GLRC-4, with two disulfide bond patterns as in GLRC-1 (C2-C18) and GLRC-3 (C6-C14), did not gain additional stability to the protease, further indicating the importance of the C4-C16 disulfide bond. Second, although GLRC-2 and GLRC-8 share the medium-sized loop, they had drastically different stability. It is likely that the sequence order in the loop determines the extent of overall packing of the peptide chain. Third, the segment outside of the loop appeared to be critical for GLRC-2 stability. In particular, removal of the N-terminal segment was detrimental, leading to complete degradation of the peptide in four hours. The C-terminal segment was less important with about $50 \%$ of the peptide remaining at $4 \mathrm{hrs}$. This is in contrast to the importance of the C-terminal segment for antiHIV activity. Nevertheless, it is interesting to note that GLRC-2, which demonstrated the greatest activity against HIV-1 (Table 1), was also the most stable to protease compared to the other GLRC peptides (Figure 2).

\section{Discussion}

In order to identify AMP candidates as lead products for the development of topical microbicides, we have continued our efforts to search for potent anti-HIV peptides. In our previous studies $[13,19]$, different engineering strategies were adopted. These included database screening, sequence shuffling, and database-aided antiviral activity

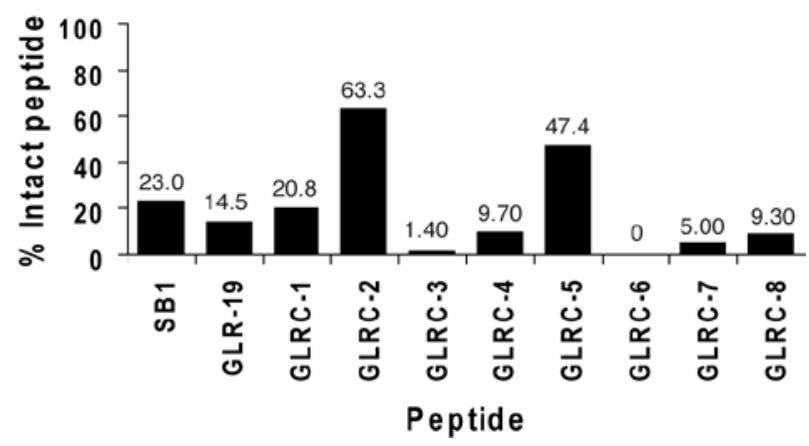

Figure 2: Stability comparison of the de novo designed GLRC peptides after incubation with chymotrypsin at $370 \mathrm{C}$ for $4 \mathrm{hrs}$ (see Methods). Both SB1 and GLR-19 are included for comparison. The percentages of uncleaved peptides are represented by the height of the bars and also indicated at the top of the bars. For example, GLRC- 5 had $\sim 50 \%$ intact peptide remaining after 4 hrs. enhancement [26]. After activity enhancement, several anti-HIV peptides in the helical family were obtained with TIs $>20$. The current study identified a $\beta$-hairpin peptide SB2 with a TI $=30$ by using the same Lys-to-Arg mutational strategy [19]. It seemed that this strategy might be applicable to the improvement of anti-HIV activity of AMPs with different structural scaffolds.

The establishment of the antimicrobial peptide database facilitated bioinformatic analysis of natural AMPs from a defined family or source $[17,18]$. For example, the frequently occurring residues from amphibian AMPs were identified as G, L, A, and K. The peptide, GLK19 (Table 1), was previously de novo designed using only G, L, and $\mathrm{K}$ residues. GLK-19 was found to be active against Gram-negative E. coli but not Gram-positive Staphylococcus aureus [17]. Because only AMPs with antiviral activity are known to have an $\mathrm{R} / \mathrm{K}$ ratio greater than one in our database, GLR-19 was designed by converting all lysines to arginines. While GLK-19 showed no HIV-1 inhibitory activity at a peptide concentration of $100 \mu \mathrm{g} / \mathrm{ml}$, GLR-19 was found to be active against both HIV-1 [19] and HSV-2 (Table 1). In this study, we have designed a series of peptides with loop structures based on the linear peptide GLR-19 (Figure 1). We demonstrated that the antiHIV activity of the peptide was also dependent on the loop size. While GLRC-2 with a medium 13-residue loop was the most potent against HIV-1, GLRC-3 with a small 9-residue loop was the most active against E. coli. It is evident that the optimal design of an antimicrobial peptide is microbe dependent.

Because these de novo designed peptides have a relatively simple amino acid composition, we also investigated possible structureactivity correlations. In the case of E. coli, there was no clear correlation between peptide activity and any physical parameters listed in Table 2, including net charge, the number of Lys or Arg, percentage of hydrophobic residues, Boman index [18] (or protein binding potential [3], and HPLC retention time (hydrophobicity). For the GLRC peptides, however, we noticed that GLRC-7, with a minimal number of positively charged residues, was the least active against Gram-negative E. coli, whereas GLRC-6, with the highest hydrophobic content $(50 \%)$, is the only peptide that was moderately active against Gram-positive B. subtilis (Table 2). These observations are in line with the current knowledge on AMPs in general [26]. It was also difficult to find any correlation between anti-HIV activity and these peptide properties. These observations indicate that multiple factors determine the biological activity of these AMPs. However, for the three most active GLRC peptides, their HIV inhibitory activities were found to be proportional to hydrophobicity as measured by HPLC (Tables 1 \& 2). In particular, the higher activity of GLRC-2 corresponded with its higher hydrophobicity, although this correlation could not be extended to all the peptides in Table 1. Craik and colleagues noticed that, among the HIV inhibitory plant cyclotides, cycloviolacin Y5, isolated from the traditional Chinese herb Viola yedoensis, was the most hydrophobic and also the most potent [27]. They proposed that cycloviolacin Y5 acted on viral membranes. Whether GLRC-2 acts by the same mechanism remains to be established. Interestingly, GLRC-2 also showed higher stability to the action of chymotrypsin compared to the other members of the GLRC peptide family as well as to its linear template GLR-19 (Figure 2).

In summary, we have demonstrated the enhancement of anti-HIV activity of linear AMPs that tend to form amphipathic helical structures using the Lys-to-Arg mutational strategy [19]. We illustrated here that anti-HIV activity of SB1, a thanatin analog with a $\beta$-hairpin fold, could also be improved by the same strategy [19]. Our current study has 
Citation: Wang G, Buckheit KW, Mishra B, Lushnikova T, Buckheit RW (2011) De Novo Design of Antiviral and Antibacterial Peptides with Varying Loop Structures. J AIDS Clinic Res S2:003. doi:10.4172/2155-6113.S2-003

expanded our knowledge of the relationship between AMP structure and anti-HIV activity. In addition to peptide length, sequence order, charge, amino acid type, and structure [13,19], peptide scaffolds or folds also play a pivotal role (Tables $1 \& 2$ ). Evaluation of peptides with varying scaffolds is important because of their different susceptibilities to proteases (Figure 2). This study led to the discovery of a novel peptide GLRC-2 that demonstrated both greater anti-HIV activity and greater stability to chymotrypsin over the GLR-19 template. These results should be helpful for our future design of highly potent antiHIV peptides with desired stability. In addition, our studies may also contribute to the identification of novel antimicrobial agents with activity against other viral and bacterial pathogens.

\section{Acknowledgements}

This study is supported by grants R21AI082689 (RWB and GW) and, in part, R56AI081975 (GW) from the National Institutes of Health.

\section{References}

1. Buckheit RW (2001) Non-nucleoside reverse transcriptase inhibitors: perspectives on novel therapeutic compounds and strategies for the treatment of HIV infection. Expert Opin Investig Drugs 10: 1423-1442.

2. Turpin JA (2002) Considerations and development of topical microbicides to inhibit the sexual transmission of HIV. Expert Opin Investig Drugs 11: 10771097.

3. Boman HG (2003) Antibacterial peptides: basic facts and emerging concepts. J Intern Med 254: 197-215.

4. Jenssen H, Hamill P, Hancock RE (2006) Peptide antimicrobial agents. Clin Microbiol Rev 19:491-511.

5. Lehrer RI (2007) Multispecific myeloid defensins. Curr Opin Hematol 14: 16-21.

6. Zasloff M (2002) Antimicrobial peptides of multicellullar organisms. Nature 415: 359-365.

7. Chinchar VG, Bryan L, Silphadaung U, Noga E, Wade D, et al. (2004) Inactivation of viruses infecting ectothermic animals by amphibian and piscine antimicrobial peptides. Virology 323: 268-275.

8. VanCompernolle SE, Taylor RJ, Oswald-Richter K, Jiang J, Youree BE, et al. (2005) Antimicrobial peptides from amphibian skin potently inhibit human immunodeficiency virus infection and transfer of virus from dendritic cells to $T$ cells. J Virol 79: 11598-11606.

9. Wachinger M, Klenschmidt A, Winder D, von Pechmann N, Ludvigsen A, et al. (1998) Antimicrobial peptides melittin and cecropin inhibit replication of human immunodeficiency virus 1 by suppressing viral gene expression. J Gen Virol 79: $731-740$

10. Robinson WE, McDougall B, Tran D, Selsted ME (1998) Anti-HIV-1 activity of indolicidin, an antimicrobial peptide from neutrophils. J Leuko Biol 63: 94-100.

11. Penberthy WT, Chari S, Cole AL, Cole AM (2011) Retrocyclins and their activity against HIV-1. Cell Mol Life Sci 68: 2231-2242.

12. Bergman P, Walter-Jallow L, Broliden K, Agerberth B, Soderlund L (2007) The antimicrobial peptide LL-37 inhibits HIV-1 replication. Curr HIV Res 5: 410-415.

13. Wang G, Watson KM, Buckheit RW Jr (2008) Anti-human immunodeficiency virus type 1 activities of antimicrobial peptides derived from human and bovine cathelicidins. Antimicrob Agents Chemother 52: 3438-3440.

14. Ireland DC, Wang CK, Wilson JA, Gustafson KR, Craik DJ (2008) Cyclotides as natural anti-HIV agents. Biopolymers 90: 51-60.

15. Wong JH, Legowska A, Rolka K, Ng TB, Hui M, et al. (2011) Effects of cathelicidin and its fragments on three key enzymes of HIV-1. Peptides 32: 1117-1122.

16. Beard JA, Bearden A, Striker R (2011) Vitamin D and the anti-viral state. J Clin Virol 50: 194-200.

This article was originally published in a special issue, Pharmacology of Antiretroviral Agents: HIV handled by Editor(s). Dr. Di Wu, The Children's Hospital of Philadelphia, USA
17. Wang G, Li X, Wang Z (2009) The updated antimicrobial peptide database and its application in peptide design. Nucleic Acids Res 37: D933-D937.

18. Wang Z, Wang G (2004) APD: the antimicrobial peptide database. Nucleic Acids Res 32: D590-D592.

19. Wang G, Watson KM, Peterkofsky A, Buckheit RW Jr (2010) Identification of novel human immunodeficiency virus type 1 inhibitory peptides based on the antimicrobial peptide database. Antimicrob Agents Chemother 54: 1343-1346.

20. Buckheit RW, Jr., Kinjerski TL, Fliakas-Boltz V, Russell JD, Stup TL, et al (1995) Structure-activity and cross-resistance evaluations of a series of human immunodeficiency virus type-1-specific compounds related to oxathiin carboxanilide. Antimicrob Agents Chemother 39: 2718-2727.

21. Wang G (2008) Structures of human host defense cathelicidin LL-37 and its smallest antimicrobial peptide KR-12 in lipid micelles. J Biol Chem 283: $32637-$ 32643.

22. Skerlavaj B, Gennaro R, Bagella L, Merluzzi L, Risso A, et al. (1996) Biological characterization of two novel cathelicidin-derived peptides and identification of structural requirements for their antimicrobial and cell lytic activities. J Biol Chem 271: 28375-28381.

23. Li X, Li Y, Han H, Miller DM, Wang G (2006) Solution structures of human LL37 fragments and NMR-based identification of a minimal mem brane-targeting antimicrobial and anticancer region. J Am Chem Soc 128: 5776-5785.

24. Fehlbaum P, Bulet $P$, Chernysh S, Briand JP, Roussel JP, et al. (1996) Structureactivity analysis of thanatin, a 21-residue inducible insect defense peptide with sequence homology to frog skin antimicrobial peptides. Proc Natl Acad Sci U S A 93: 1221-1225.

25. Mandard N, Sodano P, Labbe H, Bonmatin JM, Bulet P, et al. (1998) Solution structure of thanatin, a potent bactericidal and fungicidal insect peptide, determined from proton two-dimensional nuclear magnetic resonance data. Eur J Biochem 256: 404-410.

26. Wang G (2010) Antimicrobial Peptides: Discovery, Design and Nove Therapeutic Strategies. CABI, England.

27. Wang CK, Colgrave ML, Gustafson KR, Ireland DC, Goransson U, et al. (2008) Anti-HIV cyclotides from the Chinese medicinal herb Viola yedoensis. J N at Prod 71: 47-52. 